\title{
Towards Supporting Business Process Compliance with Policies
}

\author{
Ludmila Penicina \\ Institute of Applied Computer Systems, Riga Technical University, 1 Kalku, Riga, \\ LV-1658, Latvia \\ \{ludmila.penicina\} ertu. IV
}

\begin{abstract}
This paper discusses early findings of the research in progress to create an approach to support an organization in bridging the gap between existing business processes and policies. Business processes are valuable assets of any organization, and business process modelling has become the key activity for capturing and analysing business processes. However, advances in technology, growing expectation of openness by research funders, competition, regulations in IT security and privacy, and overall economic situation facilitate emergence of new policies, and urge enterprises to change their business processes to be compliant with the new requirements. The goal of the research is to propose the approach for closing the gap between business process models and legal states of business objects described in policies by means of using Bunge-Wand-Weber model. The approach includes means for explicit definition of legal and illegal state spaces of business objects in (1) policies, and (2) as-is business process models, and compliance checking between state spaces of (1) and (2) to indicate the gap. It is an initial input for building to-be business process models that are complaint with newly imposed policies. As a running example to illustrate the approach a publishing business process of a scholar journal is used. New policies from research funders require Open Access (OA) to all outputs from publiclyfunded research, and business processes of publishing scholar journals require changes.
\end{abstract}

Keywords: Business process modelling, BWW model, BPMN, States, Compliance.

\section{Introduction}

Business processes are valuable assets of any organization. In organizations business process modelling has become the main activity for capturing, analysing, and improving business processes. At the same time there is an increased pressure on organizations to guarantee compliance of their business processes with various legislative and regulatory requirements, other externally imposed constraints, and other policies [1] (further in the text - policies). One domain where new policies are actively adopted is research; e.g., Open Access and Open Data policies (further in the text - OA policies) are introduced to facilitate the transition to Open Science. In the EU member countries introduction of OA policies are mainly based on the European Commission's Recommendation to Member States of July 2012 that they develop and implement 
policies to ensure OA to all outputs from publicly-funded research [2]. As a result funders and research institutions have introduced new expectations and requirements, and organisations (e.g., scholar publishers) must have in place services and resources to allow compliance with funder policies [3]. According to [4] academic community will experience rapid changes in the way research is conducted, published, and results are shared. Both policies to enable OA to publications and, more recently, to research data are commonplace at European universities and around the globe, however there are other ingredients to Open Science: such as Open Reviewing and Open Software [4]. Based on that it can be predicted that new policies facilitating Open Science will be introduced in the near future, e.g., as Open Research Data pilot was introduced in Horizon 2020 [5], and organizations will have to provide compliance by introducing changes to the existing business processes.

On the other hand, nowadays organizations employ industry modelling standards like Business Process Model and Notation (BPMN) and ArchiMate to understand and improve business processes. BPMN is the de-facto standard for representing in a very expressive graphical way the processes occurring in virtually every kind of organizations [6]. However, BPMN has its limitations when it comes to modelling other aspects of organizations such as organizational structure and roles, functional breakdowns, data, strategy, business rules, and technical systems [7]. Information about Enterprise Architecture (EA) is needed to create real-world business process models. To provide a uniform representation for diagrams that describe EA, ArchiMate modelling language has been developed [8]. The core of ArchiMate language consists of three main types of elements: active structure elements (subjects), behaviour elements, and passive structure elements (objects).

Business process modelling comprises two aspects - the control-flow perspective and data-flow perspective [9]. Control-flow perspective defines possible execution paths of a business process, while data-flow perspective represents how business objects are manipulated and change states during a process. Control flow perspective is represented in business process models using BPMN. Data in business process models are usually declared in terms of business objects (physical or virtual). Business objects and subjects are represented in EA models using ArchiMate (active and passive structure). Policies impose legal (further in the text also lawful) states of business objects. The previous research has shown that BPMN lack in ability to describe flow of business objects in business process models, and explicitly declare states of business objects and state transition laws imposed by regulations (see [10], [11], and [12]). This gap hinders compliance of business processes with policies because lawful and unlawful states of business objects are not explicitly defined in business process models, models might contain meaningless states, since a set of conceivable states is not depicted, and, as the result, business process models do not represent real-world processes and can lead to business process incompliance with policies. Also, since BPMN proclaims to be directly executable, omitting states and state transition laws may hinder correct automated execution.

The goal of this research is to propose an approach to support organizations in checking compliance of existing business processes against new policies by means of Bunge-Wand-Weber model. Wand and Weber [13] built a set of models for the evaluation of modelling techniques based on an upper ontology defined by Bunge [14]. They extended Bunge's ontology and applied it to the modelling of information systems 
(BWW model). BWW model consists of constructs present in the real world that must be represented in information systems. BWW model allows straightforwardly addressing (further in the text BWW elements are in italics): (1) states of things, (2) lawful state space and lawful event space of things, (3) conceivable state space and conceivable event space of things, (4) state law that restricts values of the properties of things to a lawful subset, and (5) lawful transformations that define which events in things are lawful. BWW model provides an explicit representation of business objects, states of business objects, and state transition laws, and allows to monitor whether a business object has assumed an unlawful state. That is the reason why in this research the BWW model is used as a framework to represent in a structured way the policy and the business process for canonical comparison of both to indicate the existing gaps between the process and the policy.

Monitoring states of business objects in business processes against policies: (1) can assist organization in compliance to ensure that organization will not violate laws and there will be no potential legal problems for the organization, and (2) can contribute to consistency in collaborative business processes and customer satisfaction. A number of studies exist that show the importance of addressing states of business objects in business process models, e.g., in [15] authors indicate the importance of object states in large engineering processes such as assembling of a car or an airplane, and according to [16] in order to achieve safe execution of a process model it must be ensured that every time a task attempts to access a business object, the object is in a certain expected state (legal state). And, since not all possible transitions of states are meaningful, restrictions on object state transitions are also required. In this paper the author intentionally uses the term "business objects" and not "data objects", since active structure elements (such as actors or application components) are also capable of assuming a state which can be illegal and should be also monitored.

The research methodology is a design science method using deductive research approach. The validation of the proposed solution is out of the scope of this paper. It will be conducted using Delphi estimation method combining expert judgement. Delphi estimation will include individual estimates, sharing the estimates with experts, and having several rounds until consensus is reached.

The paper is structured as follows. In Section 2 the related work is outlined. In Section 3 the proposed approach is described. The example of applying the proposed approach is outlined in Section 4. Brief conclusions are presented in Section 5.

\section{Related Works}

The lack of consistent theoretical foundation for building information systems urged Wand and Weber to build a set of models for the evaluation of modelling techniques [13]. Wand and Weber have extended the ontology presented by Mario Bunge [14] and developed a formal foundation called BWW model for modelling information systems. Elements in BWW model (in the text shown in italics) can be organized in the following groups (adapted from [17]): 
1. Thing - including Properties, Classes and Kinds of Things. Thing is an elementary unit in BWW. Things possess Properties, which defines States of a Thing. Things can belong to Classes or Kinds depending on a number of common Properties. A Thing can act on another Thing if its existence affects the History of the other Thing. Things are coupled if one Things acts on another.

2. State of Thing - Properties of Things define their States. State Law restricts Values of Properties of Things. Conceivable State Space is a set of all States a Thing can assume. Lawful State Space defines States that comply with State Law. Stable State is a State in which a Thing or a System will remain unless forced to change by a Thing in the System Environment. Unstable State is a State that will be changed into another State by the Transformations in the System. History is the chronologically-ordered States of a Thing.

3. Transformation - transformation between States of Things. Transformation is a mapping from one State to another. Lawful Transformation defines which Events in a Thing are lawful.

4. Event - event is a change in State of a Thing. Conceivable Event Space is a set of all Events that can occur to a Thing. Lawful Event Space is a set of all Events that are lawful to a Thing. Events can be Internal Events and External Events. Events can be Well-Defined - an Event in which the subsequent State can be predicted - or Poorly-Defined - an Event in which the subsequent State cannot be predicted.

5. System - a set of coupled Things. System Composition are Things in the System. System Environment is Things outside the System interacting with the System. System Structure is a set of couplings that exists among Things. Subsystem is a System whose composition and structure is a subset of the composition and structure of another System. System Decomposition is a set of Subsystems. Level Structure is an alignment of the subsystems.

This paper continues the research presented in [11] and [12] where the evaluation of BPMN and ArchiMate against BWW model was presented. Majority of BPMN and ArchiMate core elements can be mapped to BWW constructs. However, it is necessary to supplement BPMN and ArchiMate modelling languages with the missing elements in order to be able to maintain a set of object states in business process models. In BPMN and ArchiMate there is no explicit representation for object's State, Conceivable State Space, Lawful State Space, State Law, Conceivable Event Space, Lawful Event Space, and History - the resulting BPMN and ArchiMate models may be irrelevant and modellers may need to incorporate additional modelling techniques to overcome these defects. It may be impossible to detect from BPMN and ArchiMate models which states should be expected to occur and which states can occur but are illegal (unlawful). Another important aspect is lacking of element History which chronologically describes state changes of business objects. This deficiency can lead to problems regarding maintaining system's log and recovery.

The authors of [5] propose a notion of "weak conformance" which checks conformance of a process model with respect to data objects. This notion can be used to tell whether in every execution of a process model each time a task needs to access a data object in a particular state, it is ensured that the data object is in the expected state or can reach the expected state and, hence, the process model can achieve its goals. 
In [18] authors identify that consistency between business process models and object life cycle is required, however, their relation is not well understood. Authors clarify this relation and propose an approach to establish the required consistency by explicitly defining object states in business process models and then generating life cycles for each object type in the process. The authors of [18] indicate that object life cycle modelling is valuable at the business level. However, we propose to consider states of objects also at the application and technology levels of enterprise architecture since objects can be hidden and specified in sub-process structures at different levels of an enterprise. The authors of [19] use object life cycle as a common means for explicitly modelling allowed state transitions of an object during its existence and propose a technique for generating a compliant business process model from a set of given reference object life cycles. The notion of a "legal state" is also mentioned in [20] where authors indicate that the representation of legal states in a model of a trade procedure is essential because organizations should be able to derive their obligations, rights, and duties at each point during the execution of the trade procedure and propose to annotate the states in Petri nets.

In [1] authors investigate the use of temporal deontic assignments on activities as a means to declaratively capture the control-flow semantics that reside in business regulations and business policies. In object-oriented paradigm, state machines are extensively used for representation of states of objects [21]. In [22] the authors propose logic based formalism for describing the semantics of business contracts and the semantics of compliance checking procedures and close the gap between business processes and business contracts.

This research differs from the related work in that it uses BWW model as a missing part or a bridge to close the gap between: (1) legal states represented in policies, (2) BPMN business process models, (3) active and passive structure elements represented in ArchiMate EA models (business objects and subjects).

\section{The Proposed Approach}

The proposed approach requires the following prerequisites:

1. There is a policy describing legal and illegal states of business objects that an organization must be complaint with.

2. There is a BPMN business process model that needs to be monitored against the policy to indicate the gap.

3. There is an ArchiMate EA model that describes business objects and subjects (active and passive structure elements) depicted in the BPMN business process model.

4. There is an expert from the represented business domain using the approach that is familiar with BPMN, ArchiMate and BWW model (further in the text - the modeller).

The proposed approach includes the following steps (see Figure 1): 
1. Structure a BWW model of a policy using a questionnaire - the input to the activity is an existing policy in a textual form. The questionnaire is presented to the modeller online in the form of questions to answer. The questionnaire is built specifically to answer the questions about the policy in the context of the BWW model to be able to construct the BWW model automatically with the values of the BWW model elements recorded against questions. The output of the activity is the constructed BWW model of the policy. The BWW model of the policy is constructed in a canonical way by means of an XML document. Below are examples of the questions from the questionnaire:

a. How many Things the policy describes?

b. What Things are passive elements?

c. What Things are active elements?

d. Describe Properties of each Thing (Property name and Property value).

e. Describe State Law of each Thing (Values of the Properties that are lawful).

f. Describe Lawful States based on the State Law.

2. Structure a BWW model from an as-is BPMN business process - the input to the activity is the as-is BPMN business process model that describes the existing business process. The purpose of the approach is to check the existing business process model against the policy to indicate the gap. This step uses the BPMN model to construct the BWW model to be able to compare it with the BWW model of the policy. This step uses the mapping of BPMN to the BWW model presented in [17] and [21] to construct the BWW model from the BPMN business process model. The output of the activity is the BWW model of the BPMN business process model.

3. Structure a BWW model of an as-is ArchiMate EA model - the input to the activity is the as-is ArchiMate EA model that describes the existing enterprise architecture that refines the existing BPMN business process model. This step uses the ArchiMate model to construct the BWW model to be able to compare it with the BWW model of the policy. This step uses the mapping of ArchiMate to the BWW model presented in [12] and [21] to construct the BWW model from the ArchiMate model. The output of the activity is the BWW model of the ArchiMate EA model.

4. Merge the BWW models constructed from the BPMN and ArchiMate models into one BWW model - the input to the activity are both BWW models constructed from the BPMN business process model and the ArchiMate EA model. The BWW models are represented as XML documents and merged into one XML document to represent one BWW model that will be compared with the BWW model of the policy.

5. Compare the BWW model of the policy against the BWW model of the existing business process models - two canonical representations of the BWW models are compared to indicate the gap between the existing business process models and the policy. The BWW models are represented as XML documents and two XML documents are compared to indicate the differences between the two. The gap represents elements that are missing in the existing business process models compared to the policy. If the gap exists, this means that the 
business process models are not compliant with the policy and might contain illegal business objects states. The gap is represented as a set of differences between two XML documents.

6. Step 6 and 7 are outside of the scope of the approach, however these steps are recommended for organizations since the goal of the compliance checking is to build a to-be business process model that is compliant with the policy and describes all the necessary business objects and states represented in the policy. Step 6 is performed by the modeller to analyse the indicated gap as an initial step to construct the to-be business process model. Step 7 is creating the to-be business process model (BPMN and ArchiMate models). The to-be business process model can be monitored against the policy using the previous steps.

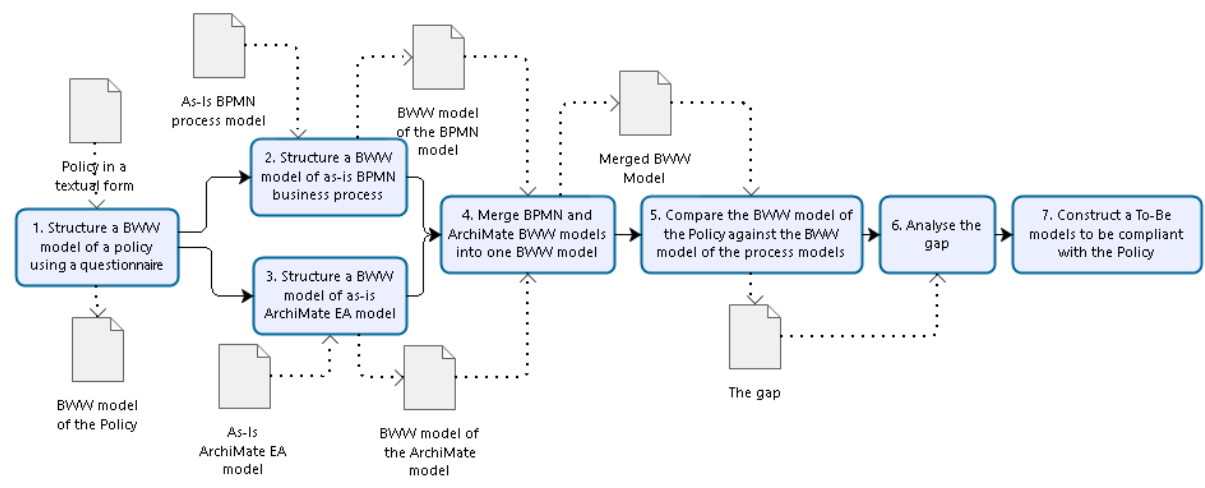

Figure 1: The steps of the proposed approach.

The construction of the BWW model from BPMN and ArchiMate models includes the following:

1. Explicitly defining Things from the models - both active and passive structure elements.

2. Explicitly defining Properties of Things (business objects and subjects) using formal definitions presented in [22] and indicating whether business object is an input or output parameter of an activity.

3. Explicitly defining Conceivable State Space based on the Properties of the Things.

4. Explicitly defining State Law of Things and Lawful State Space of business objects and subjects based on the State Law.

Compliance can be checked during or after the execution of the business process, called compliance by detection; or compliance can be checked while modelling the business process, called compliance by design [23]. The proposed approach in this paper employs the compliance by design approach. The proposed approach for monitoring compliance of business processes with policies requires a repository-based modelling tool that accommodates BPMN, ArchiMate, and BWW. 


\section{Example}

This section describes an example to explore how the proposed approach can be applied to a scholar publisher business domain and support the publisher's needs to change its journal publishing business process to be compliant with the OA initiative. The scholar publisher presented in this section is the university press that publishes the journal of the history of medicine. Existing business process of the scholar publisher is based on a traditional printed and subscription-based publishing business model, and it is clear that the publisher does not support OA. However, a deeper analysis is needed to understand the gap between the OA policy and the existing business process models. The proposed approach in this paper can be used to indicate the gap between the OA policy and the existing publishing process.

The goal of the scholar publisher is to change its publishing business process to become more visible and discoverable online, and to improve bibliometric indicators, e.g., citations. Also the research published in the journal is publicly-funded and according to the European Commission guidelines must be OA [2].

To achieve the goal the editorial team of the scholar publisher has made a decision to launch a project for inclusion in the Directory of Open Access Journals (DOAJ) [24]. DOAJ is a community-curated list of open access journals and aims to be the starting point for all information searches for quality, peer reviewed open access materials [24]. To be included in the DOAJ any publisher must be compliant with the basic requirements for inclusion in DOAJ. These requirements are available online [25], here are some examples of the DOAJ requirements:

1. All content (full texts of journal articles) should be available for free and be Open Access without delay (i.e. no embargo period).

2. User registration is not acceptable and journals requiring users to register to read full text will not be accepted.

3. All articles must have a publication date. DOAJ encourages the use of unique article identifiers, such as the DOI.

4. DOAJ considers the application of a Creative Commons license [26], or its equivalent, as the best practice.

In the context of this paper these requirements are considered as a policy that the publisher's business processes must be compliant with. Now it is possible to proceed to the first step of the approach - to make a canonical description of this policy based on the BWW model. The constructed BWW model will be used as a framework to monitor compliance of the business process. To construct the BWW model, the formal definitions of the BWW model are used described in [22]. Below (see Table 1 and Table 2) is presented a fragment of BWW model of the DOAJ inclusion policy. Table 1 shows Things and all possible Properties of Things described in the DOAJ policy, and also the Property "Type of Thing" is added for the purpose of differentiating active and passive structure elements based on ArchiMate. A Thing is the elementary unit in the BWW model. Things possess Properties. A Property is modelled via a function that maps the Thing into some value. Table 2 shows State Law and Lawful State Space based on the State Law. A State Law restricts the values of the Properties of a Thing to a subset that is deemed lawful, e.g., an article cannot be an active type of thing. Lawful State Space is the set of States of a Thing that comply with State Law of the Thing. 
According to the approach, the BWW model of the policy can be represented in a formal way, e.g., as XML document, see Code Fragment 1 of the XML code below.

Table 1: The BWW model of the DOAJ inclusion policy (1)

\begin{tabular}{|c|c|c|c|c|}
\hline \multirow{2}{*}{ Thing } & \multicolumn{4}{|c|}{ Properties } \\
\hline & Property & & Values & \\
\hline \multirow{8}{*}{ Article } & Type of Thing & Active & Passive & \\
\hline & Full text OA? & Yes & No & \\
\hline & Separate URL per article & Yes & No & \\
\hline & Publication Date & Year & Date & Not available \\
\hline & Review type & Not available & Peer review & Editorial \\
\hline & Review date & Not available & Date & \\
\hline & Copyright and licensing & Creative Commons & Other & \\
\hline & Unique Identifier & DOI & Other & Not available \\
\hline \multirow{6}{*}{ Full Texts } & Type of Thing & Active & Passive & \\
\hline & Open Access & Yes & No & \\
\hline & Embargo & Yes & No & \\
\hline & Price & For free & Charges apply & \\
\hline & $\begin{array}{l}\text { Copyright and licensing } \\
\text { information }\end{array}$ & Embedded & Not embedded & \\
\hline & Format & PDF & HTML & other \\
\hline \multirow[b]{2}{*}{ Readers } & Type of Thing & Active & Passive & \\
\hline & $\begin{array}{l}\text { Need to register to read } \\
\text { full texts? }\end{array}$ & Yes & No & \\
\hline
\end{tabular}

Table 2: The BWW model of the DOAJ inclusion policy (2)

\begin{tabular}{|c|c|c|c|}
\hline Thing & Property & State Law & Lawful State Space \\
\hline \multirow{10}{*}{ Article } & Type of Thing & Passive & \\
\hline & Full text OA? & Yes & Full Texts are OA \\
\hline & Separate URL per article & Yes & URL per Article \\
\hline & Publication Date & Year, Date & $\begin{array}{l}\text { Publication year or date is } \\
\text { available }\end{array}$ \\
\hline & \multirow{2}{*}{ Review type } & Peer review & Reviewed by peer review \\
\hline & & Editorial & Reviewed by editorial review \\
\hline & Review date & Date & \\
\hline & Copyright and licensing & Creative Commons & $\begin{array}{l}\text { Copyright and licensing } \\
\text { information available }\end{array}$ \\
\hline & \multirow{2}{*}{ Unique Identifier } & DOI & DOI unique identifier \\
\hline & & Other & Other unique identifier \\
\hline \multirow{7}{*}{ Full Texts } & Type of Thing & Passive & \\
\hline & Open Access & Yes & Full texts OA \\
\hline & Embargo & No & Full texts are not embargoed \\
\hline & Price & For free & Full texts are free \\
\hline & $\begin{array}{l}\text { Copyright and licensing } \\
\text { information }\end{array}$ & Embedded & $\begin{array}{l}\text { Copyright and licensing info } \\
\text { embedded in full text }\end{array}$ \\
\hline & \multirow{2}{*}{ Format } & PDF & Full texts are PDFs \\
\hline & & HTML & Full texts are HTML \\
\hline \multirow[b]{2}{*}{ Readers } & Type of Thing & Active & \\
\hline & $\begin{array}{l}\text { Need to register to read } \\
\text { full texts? }\end{array}$ & No & $\begin{array}{l}\text { Not asked to register to access } \\
\text { full texts }\end{array}$ \\
\hline
\end{tabular}

Code Fragment 1: The fragment of the XML code of the DOAJ policy BWW model

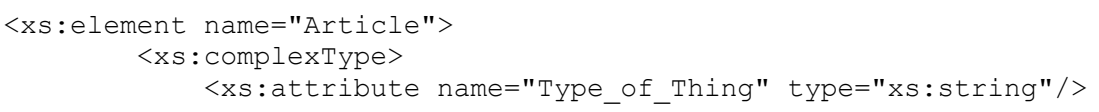




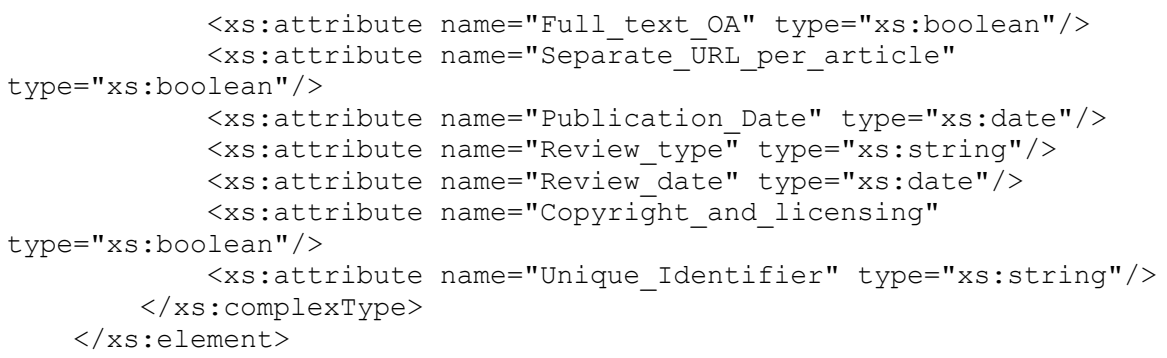

The next step of the approach is to construct a BWW model from the existing BPMN process model based on the BPMN-BWW mapping presented in [17] and [21]. A fragment of the existing publishing business process BPMN model is depicted in Figure 2. The existing ArchiMate EA model is presented in Figure 3. The BWW models of both models are constructed based on the previous work described in [17] and [21].

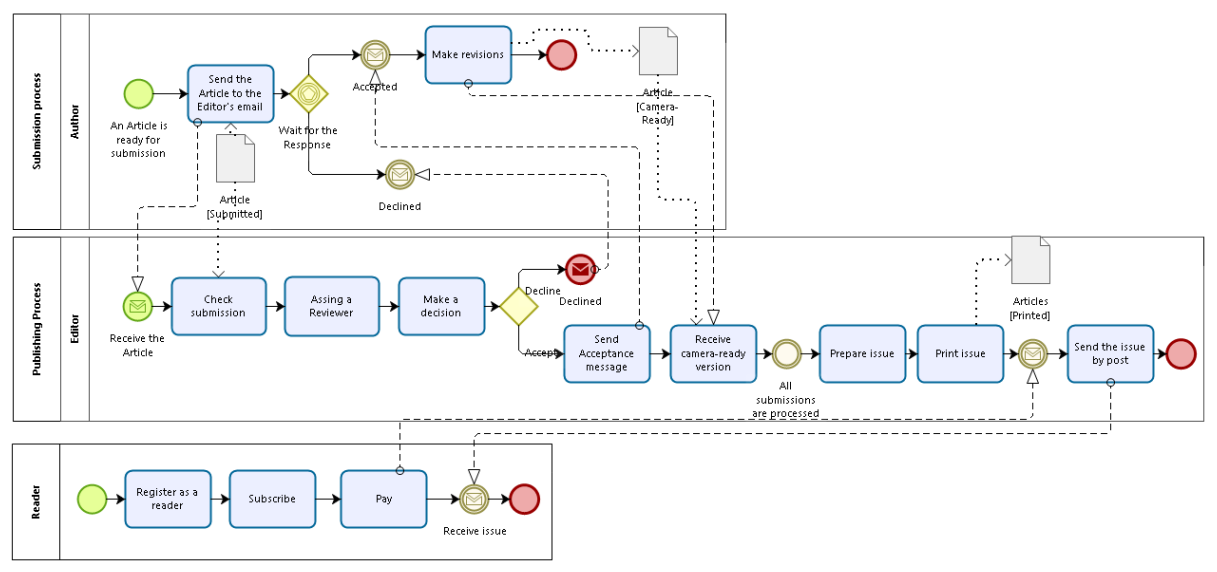

Figure 2: The fragment of the as-is BPMN business process model of the publisher.

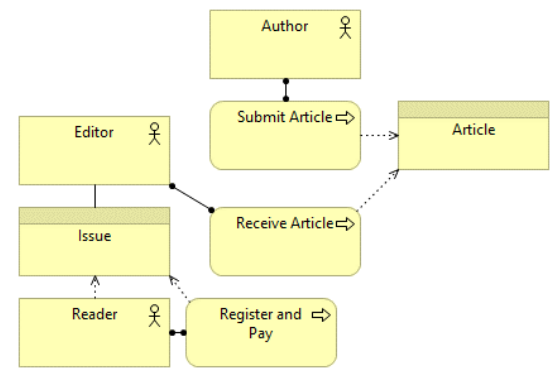

Figure 3: The fragment of the ArchiMate model of the publisher.

A fragment of the BWW model from both models are presented below (see Table $3)$. 
Table 3: The fragment of the BWW model of the existing models.

\begin{tabular}{|l|l|l|l|}
\hline Thing & \multicolumn{1}{|c|}{ Property } & State Law & \multicolumn{1}{c|}{ Lawful State Space } \\
\hline \multirow{5}{*}{ Article } & Type of Thing & Passive & \\
\cline { 2 - 4 } & Submitted & Yes & Article submitted \\
\cline { 2 - 4 } & Checked by Editor & Yes & Article checked by Editor \\
\cline { 2 - 4 } & Reviewer assigned & Yes & Reviewer assigned \\
\cline { 2 - 4 } & Reviewed & Yes & Reviewed by peer review \\
\cline { 2 - 4 } & Review date & Date & \\
\cline { 2 - 4 } & Decision made & Accepted & Article accepted \\
\cline { 2 - 4 } & Printed & Declined & Article declined \\
\cline { 2 - 4 } & Publication date & Yes & Article printed \\
\hline \multirow{5}{*}{ Issue } & Type of Thing & Date & Publication date is available \\
\cline { 2 - 4 } & Prepared & Yes & Issue prepared \\
\cline { 2 - 4 } & Printed & Yes & Issue printed \\
\cline { 2 - 4 } & Sent by post & Yes & Issue sent \\
\hline Editor & Type of Thing & Active & \\
\hline \multirow{5}{*}{ Readers } & Type of Thing & Active & \\
\cline { 2 - 4 } & $\begin{array}{l}\text { Need to register to read full } \\
\text { texts? }\end{array}$ & Yes & Reader is registered \\
\cline { 2 - 4 } & Need to pay to read full texts? & Yes & Reader has paid \\
\hline
\end{tabular}

The next step is to compare two canonical descriptions of the constructed BWW models to indicate the gap between the policy (Table 1, Table 2) and the existing business process models (Table 3). Below is presented a fragment of the comparison between lawful states of the policy and lawful states depicted in the existing models (comparison between Table 2 and Table 3 is presented, see Table 4).

Table 4: The fragment of the comparison between the BWW models.

\begin{tabular}{|l|l|}
\hline \multicolumn{1}{|c|}{$\begin{array}{c}\text { Lawful State Space from the } \\
\text { Policy }\end{array}$} & \multicolumn{1}{c|}{ Corresponding Lawful State Space from the models } \\
\hline Full Texts are OA & No corresponding lawful state description in the existing models \\
\hline URL per Article & No corresponding lawful state description in the existing models \\
\hline Publication year or date is available & Publication date is available \\
\hline Reviewed by peer review & Reviewed by peer review \\
\hline Reviewed by editorial review & No corresponding lawful state description in the existing models \\
\hline $\begin{array}{l}\text { Copyright and licensing information } \\
\text { available }\end{array}$ & No corresponding lawful state description in the existing models \\
\hline DOI unique identifier & No corresponding lawful state description in the existing models \\
\hline Other unique identifier & No corresponding lawful state description in the existing models \\
\hline Full texts OA & No corresponding lawful state description in the existing models \\
\hline Full texts are not embargoed & No corresponding lawful state description in the existing models \\
\hline Full texts are free & No corresponding lawful state description in the existing models \\
\hline $\begin{array}{l}\text { Copyright and licensing info } \\
\text { embedded in full text }\end{array}$ & No corresponding lawful state description in the existing models \\
\hline Full texts are PDFs & No corresponding lawful state description in the existing models \\
\hline Full texts are HTML & No corresponding lawful state description in the existing models \\
\hline $\begin{array}{l}\text { Not asked to register to access full } \\
\text { texts }\end{array}$ & No corresponding lawful state description in the existing models \\
\hline
\end{tabular}

From the Table 4 it can be concluded: (1) that existing business process models are not compliant with the policy and (2) which lawful states of business objects and subjects from the policy are not represented in the existing business process models. 
This gap is an input for constructing a to-be business process model that is compliant with the policy. This example does not fully represent the proposed approach, only the main idea behind the approach is illustrated. The presented work is a research in progress.

\section{Conclusions}

This paper presents an ongoing research towards supporting organization in monitoring compliance of business processes with policies. The BWW model is used as the foundation, since it allows straightforwardly addressing the lawful and conceivable state spaces of business objects. The previous research has shown that BPMN and ArchiMate lack in ability to describe flow of business objects in business process models and explicitly declare states of business objects imposed by regulations (see [10], [11] and [12]). This gap hinders compliance of business process models with different policies. There are 6 BWW model elements that are not supported by these modelling languages, namely, State Law, Conceivable State Space, Lawful State Space, History, Conceivable Event Space, and Lawful Event Space.

This research differs from the related work in that it uses the BWW model as a missing part or a bridge to close the gap between: (1) legal states represented in policies, (2) BPMN business process models, and (3) active and passive structure elements represented in ArchiMate EA models (business objects and subjects). The proposed approach supports organization in defining a canonical representation of policies using the questionnaire that is based on the BWW model. Based on the questionnaire answers the BWW model of policy is constructed. The proposed approach includes construction of the BWW model for existing BPMN and ArchiMate models to compare it with the BWW model of the policy to indicate the gap between existing business processes and policies. Comparison between the structured representations of (1) the policy, and (2) the business process models provides organization an explicit method to see what states are missing and with what elements the existing business process must be refined in order to be compliant with the new policy. The paper describes how the proposed approach can support scholar publishers in achieving compliance with the OA policies. However, the proposed approach is developed to be universal and can be used in other business domains.

Definition of object states in business process models are especially required in datadriven processes - in any process model that is based on data and manipulates with business objects. The main contribution of the research will be a formalized solution prototype that will support organizations in facilitating monitoring of the compliance of business processes with policies.

\section{Acknowledgments}

This research is funded in part by the Latvian Council of Science grant for project No $342 / 2012$, and in part by the Latvian National research program SOPHIS under grant agreement Nr.10-4/VPP-4/11. 


\section{References}

1. Goedertier, S., Vanthienen, J.: Designing Compliant Business Processes with Obligations and Permissions. In: Business Process Management Workshops. pp. 5-14 (2006).

2. European Commission: Communication from the Commission to the European Parliament, the Council, the European Economic and Social Committee and the Committee of the Regions "Towards better access to scientific information: Boosting the benefits of public investments in researc. 1-12 (2012).

3. Fina, F., Proven, J.: Using a CRIS to Support Communication of Research: Mapping the Publication Cycle to Deposit Workflows for Data and Publications. Procedia Comput. Sci. 106, 232-238 (2017).

4. van der Aalst, W., Bichler, M., Heinzl, A.: Open Research in Business and Information Systems Engineering. Bus. Inf. Syst. Eng. 58, 375-379 (2016).

5. European Commission: Guidelines on open access to scientific publications and research data in Horizon 2020. 10 (2016).

6. Chinosi, M., Trombetta, A.: BPMN: An introduction to the standard. Comput. Stand. Interfaces. 34, 124-134 (2012).

7. Silver, B.: BPMN Method and Style with Implementer's Guide. Cody-Cassidy Press (2011).

8. Group, T.O., Forum, A.: An Introduction to ArchiMate, an Open Group Standard. (2012).

9. Weske, M.: Business Process Management. Springer Berlin Heidelberg, Berlin, Heidelberg (2012).

10. Rosemann, M., Recker, J., Indulska, M., Green, P.: A Study of the Evolution of the Representational Capabilities of Process Modeling Grammars. Adv. Inf. Syst. Eng. 447-461 (2006).

11. Penicina, L., Kirikova, M.: Towards Completeness and Lawfulness of Business Process Models. Perspect. Bus. Informatics Res. Lect. Notes Bus. Inf. Process. Vol.158, 63-77 (2013).

12. Penicina, L.: Linking BPMN, ArchiMate, and BWW: Perfect Match for Complete and Lawful Business Process Models? Short Pap. Proc. 6th IFIP WG 8.1 Work. Conf. Pract. Enterp. Model. (PoEM 2013). Vol-1023, 156-165 (2013).

13. Wand, Y., Weber, R.: On the ontological expressiveness of information systems analysis and design grammars. Inf. Syst. J. 3, 217-237 (1993).

14. Bunge, M.: Treatise on Basic Philosophy: Vol. 4: Ontology II: A World of Systems. (1979).

15. Dominic, M., Müller, D., Reichert, M., Herbst, J.: A new paradigm for the enactment and dynamic adaptation of data-driven process structures. Adv. Inf. Syst. Eng. 48-63 (2008).

16. Meyer, A., Weske, M.: Weak Conformance between Process Models and Synchronized Object Life Cycles. In: ZEUS. pp. 359-367 (2014).

17. Recker, J., Indulska, M., Rosemann, M., Green, P.: Do Process Modelling Techniques Get Better? A Comparative Ontological Analysis of BPMN. Campbell, Bruce, Underwood, Jim, Bunker, Deborah 16th Australas. Conf. Inf. Syst. (2005). 
18. Ryndina, K., Küster, J.M., Gall, H.: Consistency of Business Process Models and Object Life Cycles. In: Models in Software Engineering. pp. 80-90. Springer Berlin Heidelberg, Berlin, Heidelberg (2007).

19. Küster, J.M., Ryndina, K., Gall, H.: Generation of Business Process Models for Object Life Cycle Compliance. In: Business Process Management. pp. 165181. Springer Berlin Heidelberg, Berlin, Heidelberg (2007).

20. Bons, R.W.H., Lee, R.M., Wagenaar, R.W., Wrigley, C.D.: Modelling interorganizational trade using Documentary Petri Nets. In: Proceedings of the Twenty-Eighth Hawaii International Conference on System Sciences, vol.3. pp. 189-198. IEEE Comput. Soc. Press (1996).

21. Penicina, L., Kirikova, M.: Towards Controlling Lawful States and Events in Business Process Models. In: IEEE 16th Conference on Business Informatics (CBI 2014). pp. 15-23 (2014).

22. Penicina, L., Kirikova, M.: Towards Compliance Checking between Business Process Models and Lawful States of Objects. In: 2nd International Workshop on Ontologies and Information Systems (WOIS 2014). pp. 30-41 (2014).

23. Lohmann, N.: Compliance by Design for Artifact-Centric Business Processes. In: Information Systems. pp. 99-115 (2011).

24. Directory of Open Access Journals, https://doaj.org/about.

25. DOAJ: Publishing best practice and basic standards for inclusion, https://doaj.org/publishers\#advice.

26. Creative Commons, http://creativecommons.org/.

Penicina L. (2017) Towards Supporting Business Process Compliance with Policies. In: Johansson B., Møller C., Chaudhuri A., Sudzina F. (eds) Perspectives in Business Informatics Research. BIR 2017. Lecture Notes in Business Information Processing, vol 295. Springer, Cham. https://doi.org/10.1007/978-3-319-64930-6_7 